\section{OPEN ACCESS}

Edited by:

Weimin Ye,

Karolinska Institutet (KI), Sweden

Reviewed by:

Chutima Roomruangwong, Chulalongkorn University, Thailand

Clelia de Oliveira Lyra,

Federal University of Rio Grande do

Norte, Brazi

${ }^{*}$ Correspondence:

Peiyu Wang

wpeiyu@bjmu.edu.cn

Yumei Zhang

zhangyumei@bjmu.edu.cn

Specialty section: This article was submitted to

Nutritional Epidemiology,

a section of the journa

Frontiers in Nutrition

Received: 05 May 2021

Accepted: 30 July 2021

Published: 26 August 2021

Citation:

Yang C, Zhao A, Lan H, Ren Z, Zhang J, Szeto IM-Y, Wang $P$ and

Zhang $Y$ (2021) Association Between

Dietary Quality and Postpartum Depression in Lactating Women: A

Cross-Sectional Survey in Urban

China. Front. Nutr. 8:705353.

doi: 10.3389/fnut.2021.705353

\title{
Association Between Dietary Quality and Postpartum Depression in Lactating Women: A Cross-Sectional Survey in Urban China
}

\begin{abstract}
Chenlu Yang ${ }^{1}$, Ai Zhao ${ }^{2}$, Hanglian Lan ${ }^{3,4}$, Zhongxia Ren ${ }^{1}$, Jian Zhang ${ }^{1}$, Ignatius Man-Yau Szeto ${ }^{3,4}$, Peiyu Wang ${ }^{5 *}$ and Yumei Zhang ${ }^{1,6 *}$
\end{abstract}

${ }^{1}$ Department of Nutrition and Food Hygiene, School of Public Health, Peking University, Beijing, China, ${ }^{2}$ Vanke School of Public Health, Tsinghua University, Beijing, China, ${ }^{3}$ Inner Mongolia Dairy Technology Research Institute Co., Ltd., Hohhot, China, ${ }^{4}$ Yili Maternal and Infant Nutrition Institute, Inner Mongolia Yili Industrial Group Co., Ltd., Hohhot, China, ${ }^{5}$ Department of Social Medicine and Health Education, School of Public Health, Peking University, Beijing, China, ${ }^{6}$ Beijing Key Laboratory of Toxicological Research and Risk Assessment for Food Safety, School of Public Health, Peking University, Beijing, China

Background: Evidence on the effects of dietary quality on the risk of postpartum depression in the Chinese population is limited. This study aimed to examine the association between dietary quality and postpartum depression in Chinses lactating women.

Methods: A total of 939 participants from 10 cities were included in this analysis. A one-time 24-h dietary recall was used to obtain the data on food consumption and dietary quality was assessed based on Diet Balance Index. The Edinburgh postnatal depression scale was considered at a cutoff point of 10 to detect postpartum depression. Poisson regression models were used to explore the association of dietary quality with postpartum depression.

Results: Depressed women tended to have a more inadequate intake of vegetables and have more insufficient food variety. The median (25th, 75th) of the overall high bound score (HBS), low bound score (LBS), and diet quality distance (DQD) was 9 (5, 14), 30 $(25,37)$, and $40(34,47)$, respectively. Compared with subjects with the lowest quartile of LBS, those with the highest quartile of LBS had a higher risk of postpartum depression [adjusted prevalence ratio (aPR), 1.08; 95\% confidence interval (95\% Cl), 1.01, 1.15; P for trend, 0.043]. We also observed a significant association between DQD and postpartum depression (Q4 vs. Q1: aPR, 1.07; 95\% Cl: 1.00, 1.14; P for trend, 0.036).

Conclusion: Poor dietary quality was associated with postpartum depression in Chinese lactating women.

Keywords: diet quality, postpartum depression, diet balance index, lactating women, Chinese dietary guidelines

\section{INTRODUCTION}

Postpartum depression is a widely recognized public health concern that has important implications for the health and well-being of many new mothers, their infants, and their families $(1,2)$. Depressive symptoms may include extreme sadness, fatigue, anxiety, crying, irritability, and changes in lifestyle habits (3). A meta-analysis has reported that pooled prevalence of postpartum depression was $14.8 \%$ and showed a significant increasing trend in the last decade 
in Mainland China (4). There may be numerous mechanisms mediating the development of a typical pathophysiological signature associated with postpartum depression (5). Still, the etiology of postpartum depression has not been fully cleared. Even worse, some women may be unwilling to take pharmacological treatments, because they concern about the transmission of antidepressant medication to their infants if they are breastfeeding (6). Therefore, the efforts toward the identification of risk factors are crucial for both maternal and children's health and well-being.

Dietary quality may be a modifiable risk factor for depression because some nutritional factors may modulate the potential biological pathways related to mental disorders, such as inflammation, oxidative stress, the gut microbiome, epigenetic modifications, and neuroplasticity (7). A healthy dietary pattern, which was characterized by fruits, vegetables, whole grains, and fish, may decrease the risk of depression $(8,9)$. However, the associations of dietary quality with postpartum depression have primarily been explored in western countries (10-13), and results were inconsistent. Researches among Chinese lactating women are less. Despite the knowledge that lactating women require varied diets and increased nutrient intake, Chinese lactating women tend to be more prone to poor dietary quality, especially for the women in the puerperium. Chinese traditional beliefs about the importance of "Yin-Yang balance" affect the daily food practices of lactating women. Childbirth is believed to disturb the "Yin-Yang balance," and different food characteristics (such as "hot" and "cold") can alter the "Yin-Yang balance" in the body (14-16). Usually, the "cold" foods were related "Yin." Therefore, lactating women should avoid "cold" foods to maintain body warmth and restore maternal "Yin-Yang balance." For example, fresh fruits or vegetables, even seafood, are not highly recommended because most of these foods are considered to have "cold" properties. In addition, lactating women are encouraged to consume more certain foods, such as animal products, red sugar, even rice wine, because these foods have been traditionally regarded as beneficial to maternal health and breast milk quantity and quality. However, these dietary restrictions are not aligned with nutritional needs and may cause some common postpartum symptoms. For example, a decreased intake of fruit and vegetables and excessive sugar intake are associated with a high incidence of constipation, hemorrhoids, and oral problems (17).

The objective of the current study was to explore the potential associations between dietary quality and postpartum depression.

\section{METHODS}

\section{Participants}

The data were collected as part of the Young Investigation (YI Study), which was a cross-sectional survey on health and nutrition status of pregnant women, lactating women, young children aged 0-3 years from 2019 to 2020. Two first-tier cities (Beijing and Guangzhou), three new first-tier cities (Suzhou, Chengdu, and Shenyang), three second-tier cities (Ningbo, Lanzhou, and Nanchang), one third-tier city (Hohhot), and one fourth-tier city (Xuchang) were selected. One hospital or one maternal and child health care center was selected in each city. The target was to recruit at least 90 lactating women in each city, and lactating women were conveniently recruited according to their visiting time until the number of participants satisfied the sample size. For lactating women, the inclusion criteria were healthy women in the first year postpartum, aged between 20 and 45 years, with singleton delivery, no smoking or alcohol abuse, without mastitis or any infectious diseases, and without cardiovascular or metabolic diseases. We excluded those participants with missing data or extreme outliers for key variables under the purpose of this report. Finally, 939 participants were enrolled in the current study.

\section{Data Collection and Measures}

The investigation was conducted using face-to-face interviews. All interviewers were subject to unified training before starting the investigation.

Postpartum depression was assessed by the Edinburgh Postnatal Depression Scale (EPDS). The EPDS included 10 items using 4-point response options ranging from 0 to 3 to capture symptoms in the previous seven days. The total score ranged from 0 to 30, with higher scores indicating greater severity of depression. The cutoff scores of $\geq 10$ were used to classify nondepressed and depressed mothers, respectively, and the cutoff scores of $\geq 13$ were used to further classify mildly depressed and moderate or severe depressed mothers, respectively, among the depressed mothers $(18,19)$.

A one-time 24-h dietary recall (24HDR) was used to obtain the data on food consumption over the previous $24 \mathrm{~h}$ to the investigation. With the help of trained interviewers, participants were asked to recall all food, beverages, and condiments consumed individually over the previous $24 \mathrm{~h}$ to the investigation. Standard-sized bowls, standard-sized teaspoons, and illustrated photos of food items were shown to help participants to assess quantities (20). Total energy intake was calculated based on the Chinese Food Composition Table coupled with the nutrition information packaging (21). In China, Diet Balance Index (DBI) was recommended to assess human diet quality. It can measure the dietary quality as a whole, and reflect not only insufficient dietary intake but also excessive dietary intake $(22,23)$. DBI was established based on the Chinese dietary guidelines. It was firstly released in 2005 and has been updated twice, with DBI_16 being the current measure (24-26). DBI_16 was based on current Chinese dietary guidelines and Food Guide Pagoda (2016), and has more specific energy assignment levels that comprehensively reflect the dietary quality of the population. DBI_16 could be minor adjusted to meet the needs of different populations based on specific dietary guidelines, and a previous study has proved that adjusted DBI was a reliable tool to measure the diet quality among lactating women (27). In this study, DBI comprised eight components, namely (range of values) (1) cereals $(-12 \sim 12)$; (2) vegetables $(-6 \sim 0)$, fruits $(-6 \sim 0)$; (3) dairy ( $-6 \sim 0)$, soybean $(-6 \sim 0)$; (4) animal foods $(-4 \sim 4$ for meat and poultry, $-4 \sim 0$ for fish and shrimp, $-4 \sim 4$ for eggs); (5) empty energy food ( $0 \sim 6$ for cooking oil, $0 \sim 6$ for alcohol); (6) salt $(0 \sim 6)$; ( 7$)$ food variety $(-12 \sim 0)$; and $(8)$ water $(-12 \sim 0)$. A score of 0 for each component indicated that the recommended 
intake had been met. The positive or negative scores indicated that recommended level was exceeded or not met, respectively. The specific calculating methods of each component are given in Supplementary Table 1. After calculating the scores of each component, high bound score (HBS), low bound score (LBS), and diet quality distance (DQD) were calculated. The HBS referred to the sum of all positive scores, indicating excessive food intake (range: 0 38). The LBS referred to the sum of the absolute values of all negative scores, indicating insufficient food intake (range: $0 \sim 72$ ). DQD referred to the sum of the absolute values of both positive and negative scores, indicating imbalanced food intake (range: $0 \sim 90$ ). The larger values for HBS, LBS, and DQD indicate more inferior diet quality. HBS, LBS, and DQD were further divided into four levels (excellent or good, mild poor, moderately poor, and severe poor) according to $0 \sim 40 \%, 40 \sim 60 \%, 60 \sim 80 \%$, and $80 \sim 100 \%$ of total scores, respectively.

Essential characteristics of the lactating women were collected, including age, education, family monthly per capita income
(Chinese yuan), puerperium (within 6 weeks after delivery) or not, and primiparas or not. Weight and height were measured on the day of investigation, and body mass index (BMI) was coded into three categories: underweight or normal (BMI $<24 \mathrm{~kg} / \mathrm{m}^{2}$ ), overweight $\left(24 \leq \mathrm{BMI}<28 \mathrm{~kg} / \mathrm{m}^{2}\right)$, and obese $\left(\mathrm{BMI} \geq 28 \mathrm{~kg} / \mathrm{m}^{2}\right)$ (28). Based on the short version of the International Physical Activity Questionnaire (IPAQ), metabolic equivalent of energy (MET) hours per week were calculated, and the physical activity of participants was equally divided into three groups: Low, medium, and High (29). The status of husband or partner, and parent support were self-reported by lactating women (rarely, sometimes, or always).

\section{Statistical Analyses}

The statistical analysis was performed by and SPSS 26.0. All non-normally distributed continuous variables are presented as median (25th, 75th). All categorical variables are presented as percentages. First, chi-square tests were performed to compare

TABLE 1 | Characteristics of lactating women by depression status.

\begin{tabular}{|c|c|c|c|c|c|c|}
\hline Variables & & Total & Non-depression & Mild depression & $\begin{array}{c}\text { Moderate or } \\
\text { severe depression }\end{array}$ & $P^{a}$ \\
\hline $\mathrm{N}$ & - & 939 & 621 & 166 & 152 & - \\
\hline \multirow[t]{2}{*}{ Age (years) } & $\leq 30$ & $472(50.3)$ & $290(46.7)$ & $87(52.4)$ & $95(62.5)$ & 0.002 \\
\hline & $>30$ & $467(49.7)$ & 331 (53.3) & 79 (47.6) & 57 (37.5) & - \\
\hline \multirow[t]{2}{*}{ College or university } & No & $235(25.0)$ & 153 (24.6) & 33 (19.9) & 49 (32.2) & 0.037 \\
\hline & Yes & $704(75.0)$ & 468 (75.4) & $133(80.1)$ & $103(67.8)$ & - \\
\hline \multirow{3}{*}{$\begin{array}{l}\text { Family monthly per capita } \\
\text { income (Chinese yuan) }\end{array}$} & $<5,000$ & $424(45.2)$ & 277 (44.6) & $73(44.0)$ & $74(48.7)$ & 0.680 \\
\hline & $5,000-9,999$ & $349(37.2)$ & 239 (38.5) & $60(36.1)$ & $50(32.9)$ & - \\
\hline & $\geq 10,000$ & $166(17.7)$ & 105 (16.9) & 33 (19.9) & $28(18.4)$ & - \\
\hline \multirow[t]{2}{*}{ Puerperium } & Yes & 202 (21.5) & 143 (23.0) & 33 (19.9) & $26(17.1)$ & 0.240 \\
\hline & No & 737 (78.5) & 478 (77.0) & $133(80.1)$ & $126(82.9)$ & - \\
\hline \multirow[t]{2}{*}{ Primiparas } & Yes & $620(66.0)$ & 391 (63.0) & $113(68.1)$ & 116 (76.3) & 0.006 \\
\hline & No & $319(34.0)$ & $230(37.0)$ & $53(31.9)$ & $36(23.7)$ & - \\
\hline \multirow[t]{3}{*}{ BMI } & $\begin{array}{l}\text { Underweight or normal } \\
\text { weight }\end{array}$ & $593(63.2)$ & $390(62.8)$ & $108(65.1)$ & 95 (62.5) & 0.946 \\
\hline & Overweight & $264(28.1)$ & $174(28.0)$ & $46(27.7)$ & $44(28.9)$ & - \\
\hline & Obese & $82(8.7)$ & $57(9.2)$ & $12(7.2)$ & $13(8.6)$ & - \\
\hline \multirow[t]{3}{*}{ Physical activity } & Low & $343(36.5)$ & $226(36.4)$ & $60(36.1)$ & $57(37.5)$ & 0.978 \\
\hline & Medium & $298(31.7)$ & $201(32.4)$ & $51(30.7)$ & 46 (30.3) & - \\
\hline & High & $298(31.7)$ & 194 (31.2) & $55(33.1)$ & $49(32.2)$ & - \\
\hline \multirow[t]{3}{*}{ Husband or partner support } & Rarely & $53(5.6)$ & $33(5.3)$ & $12(7.2)$ & $8(5.3)$ & 0.003 \\
\hline & Sometimes & $211(22.5)$ & 119 (19.2) & $54(32.5)$ & $38(25.0)$ & - \\
\hline & Always & 675 (71.9) & 469 (75.5) & $100(60.2)$ & $106(69.7)$ & - \\
\hline \multirow[t]{3}{*}{ Parent support } & Rarely & $61(6.5)$ & $35(5.6)$ & $16(9.6)$ & $10(6.6)$ & 0.066 \\
\hline & Sometimes & $189(20.1)$ & $114(18.4)$ & $42(25.3)$ & $33(21.7)$ & - \\
\hline & Always & 689 (73.4) & $472(76.0)$ & $108(65.1)$ & $109(71.7)$ & - \\
\hline \multirow[t]{4}{*}{ Cities } & First-tier & 187 (19.9) & 122 (19.6) & $36(21.7)$ & $29(19.1)$ & 0.697 \\
\hline & New first-tier & 277 (29.5) & 194 (31.2) & 44 (26.5) & $39(25.7)$ & - \\
\hline & Second-tier & $283(30.1)$ & 184 (29.6) & 48 (28.9) & $51(33.6)$ & - \\
\hline & Third or fourth-tier & $192(20.4)$ & 121 (19.5) & $38(22.9)$ & $33(21.7)$ & - \\
\hline \multirow[t]{2}{*}{ Regions } & South & $467(49.7)$ & 300 (48.3) & 88 (53.0) & 79 (52.0) & 0.467 \\
\hline & North & $472(50.3)$ & $321(51.7)$ & 78 (47.0) & 73 (48.0) & - \\
\hline
\end{tabular}

${ }^{a}$ Chi-square test. 
the characteristics of lactating women by depression status. Second, Mann-Whitney $U$ or Kruskal-Wallis $H$ tests were performed to compare the HBS, LBS, and DQD by different characteristics of lactating women. Third, Spearman correlation analyses were performed to explore the associations of total scores of EPDS with HBS, LBS, and DQD. Mann-Whitney $\mathrm{U}$ tests were performed to compare each food component, HBS, LBS and DQD of lactating women by depression status. Fourth, poisson regression models were performed to explore the associations of HBS, LBS, and DQD with postpartum depression, and prevalence ratios (PRs) and their respective 95\% confidence intervals (95\%CIs) were estimated.

\section{RESULTS}

As shown in Table 1, the overall proportion of postpartum depression was $33.9 \%$, which was comprised of mild depression (17.7\%) and moderate or severe depression (16.2\%). Depressed women tended to be younger, less educated, primiparas, and with less husband or partner support.
Overall the proportion of participants meeting the recommended dietary intakes (score $=0$ ) was ranged from 0.0 to $98.9 \%$, with $0.0 \%$ for food variety, $7.8 \%$ for meat and poultry, $7.9 \%$ for dairy, $9.8 \%$ for eggs, $10.6 \%$ for vegetables, $11.0 \%$ for cereals, $18.6 \%$ for fish and shrimp, $26.0 \%$ for fruits, $28.4 \%$ for soybean, $31.1 \%$ for salt, $51.2 \%$ for water, $59.1 \%$ for cooking oil, and $98.9 \%$ for alcohol. As shown in Figure 1, the insufficient food intake of vegetables, fruits, dairy, soybean, and fish and shrimp were common. The median score of food variety was only -5 .

The median (25th, 75th) of overall HBS, LBS, and DQD was $9(5,14), 30(25,37)$, and $40(34,47)$, respectively. The sum of the proportions of HBS, LBS, and DQD in the poor level was as high as 53.7, 97.3, and 99.7\%, respectively (Figure 2). As shown in Table 2, lactating women aged $\leq 30$, with lower education level and family income tended to have higher LBS, and lactating women with lower education level and family income tended to have higher DQD.

As shown in Figure 3, depressed women tended to have a more inadequate intake of vegetables and have more insufficient

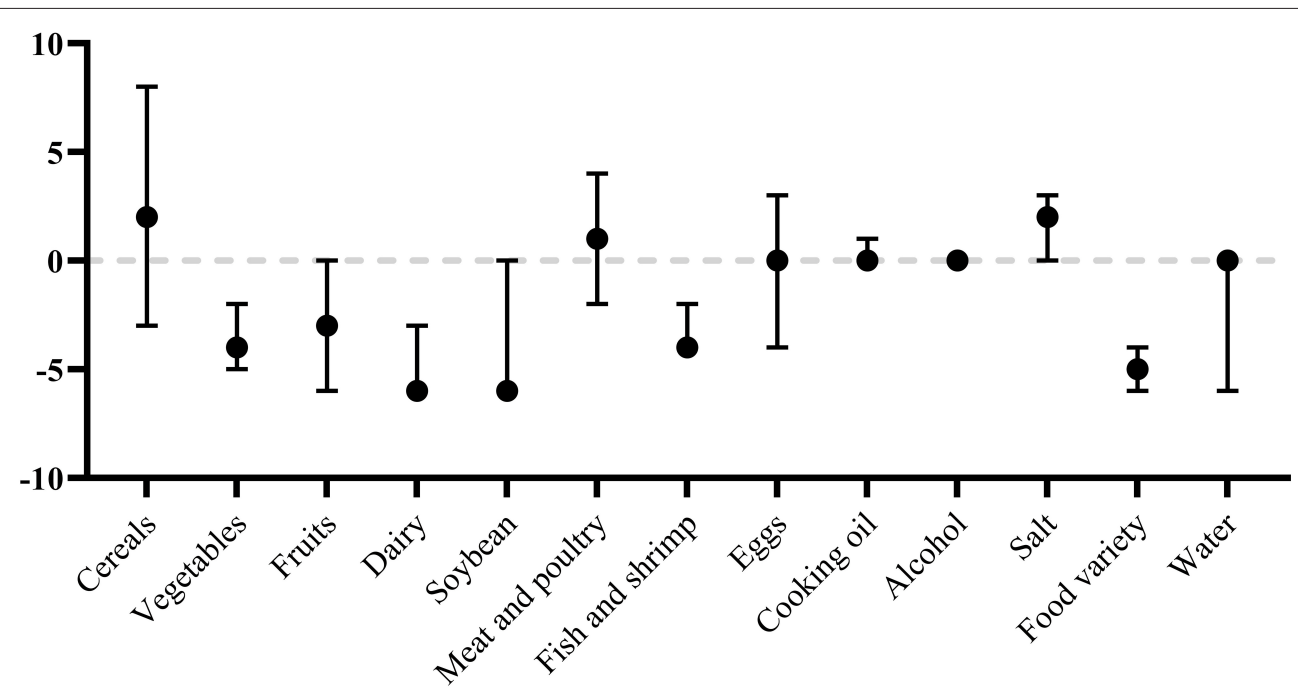

FIGURE 1 | Scores [Median (25th, 75th)] for the DBI components.

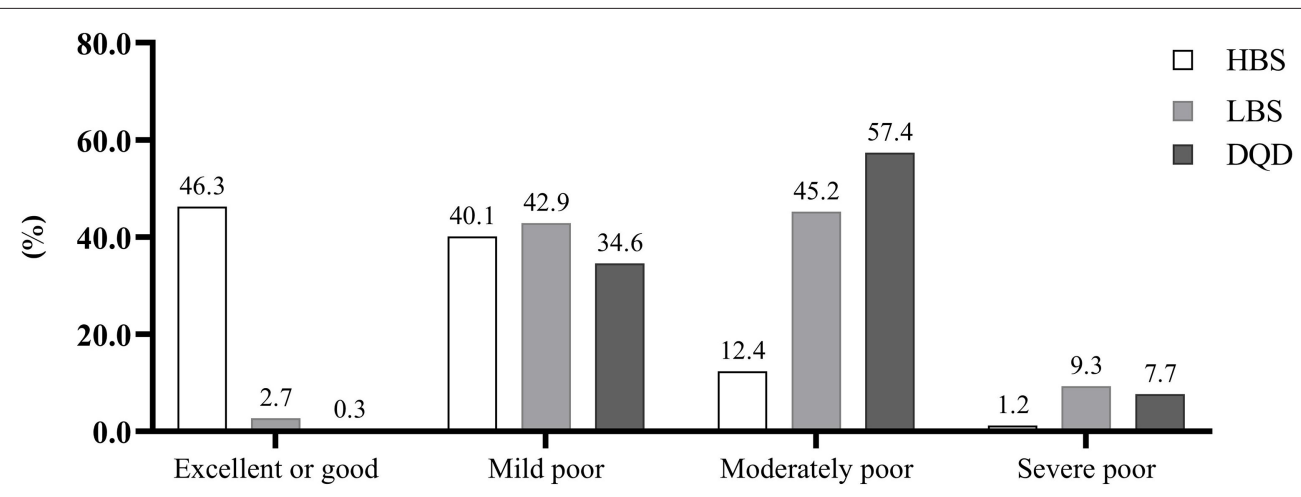

FIGURE 2 | Distributions of HBS, LBS, and DQD among lactating women. 
TABLE 2 | Comparison of HBS, LBS, and DQD [Median (25th, 75th)] by different characteristics of lactating women.

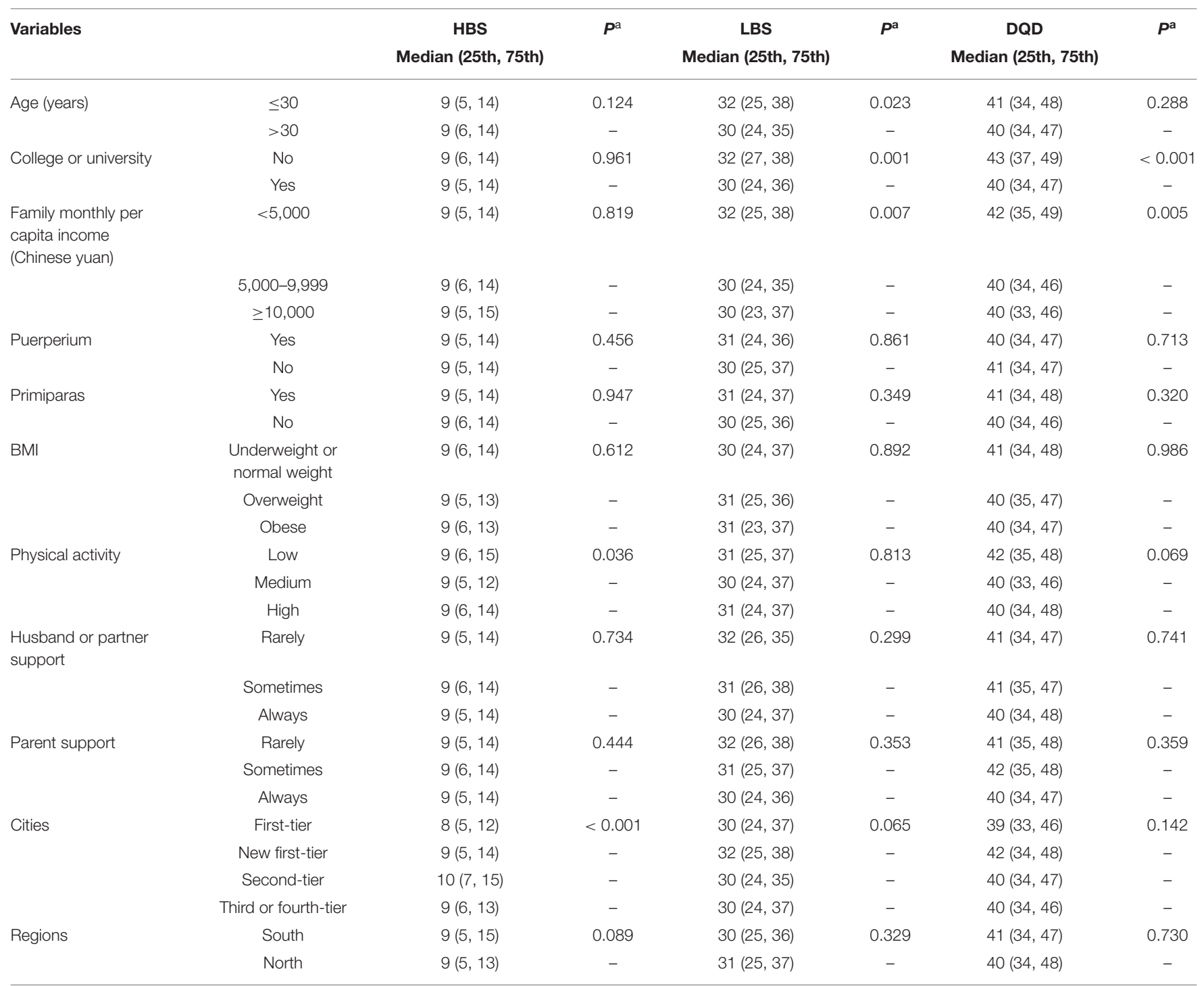

a Mann-Whitney U or Kruskal-Wallis H-test.

food variety. However, depressed mothers tended to have a slightly more moderate intake of eggs. The correlations of total scores of EPDS with HBS, LBS, and DQD were -0.007 ( $P=$ $0.939), 0.064(P=0.048)$, and 0.065 ( $P=0.047)$, respectively. As shown in Table 3, depressed women tended to have higher LBS and DQD.

As shown in Table 4, a significant association was observed between LBS and postpartum depression; in addition, a significant association was observed between DQD and postpartum depression.

\section{DISCUSSION}

To our knowledge, it is the first study to assess the association of dietary quality and postpartum depression of Chinese lactating women in the first year postpartum based on the DBI. We found that nearly one-third of Chinese lactating women in the first year postpartum suffered from depressive symptoms. Those with younger age, lower education, being primiparous, and with less support from husband or partner were more vulnerable. The dietary quality of lactating women was poor, the intake of vegetables, fruits, dairy, soybean, and fish and shrimp was insufficient, and the food variety was very limited. Depressed women had less vegetable intake and had more insufficient food variety. An imbalanced diet was associated with an increased risk of postpartum depression.

Postpartum depression is a mood disorder associated with childbirth, usually develops between a week and a month after delivery. Puerperium is considered as a period of the high prevalence of mental disorder illnesses in women (30), but in this study, no significant difference was observed between women in puerperium and after puerperium. Postpartum 
- Non-depression

- Depression

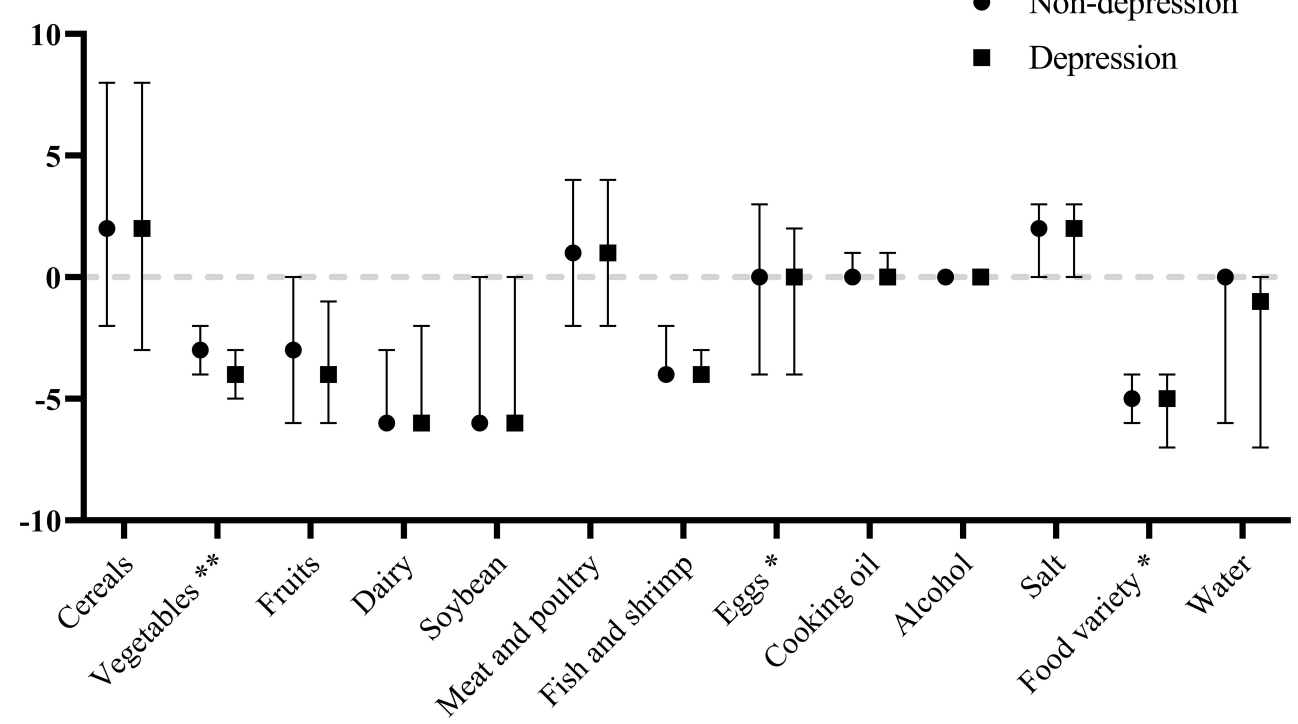

FIGURE 3 | Comparison of scores [Median (25th, 75th)] for the DBI components by depression status. ${ }^{* \star} P<0.01$; ${ }^{\star} P<0.05$.

TABLE 3 | Comparison of HBS, LBS, and DQD [Median $\left.\left(25^{\text {th }}, 75^{\text {th }}\right)\right]$ by depression status.

\begin{tabular}{lccc}
\hline & Non-depression & Depression & $\boldsymbol{P}^{\mathbf{a}}$ \\
\hline HBS & $9(5,14)$ & $9(5,14)$ & 0.323 \\
LBS & $30(24,36)$ & $31(26,39)$ & 0.010 \\
DQD & $40(34,47)$ & $42(35,49)$ & 0.025 \\
\hline
\end{tabular}

a Mann-Whitney U-test.

depression can be spontaneously alleviated within 3-6 months, but some depressed mothers still have symptoms at 1 year, with increased negative effects on both maternal and children's health and well-being (31). Some researchers suggest the assessment period of postpartum depression should be extended to 12 months postpartum (32). Further, although the National Health Commission (NHC) of China has planned that introducing screening for depression during pregnancy and childbirth to routine antenatal care and postpartum visits, one study has found that the majority of symptom-positive women during the perinatal period do not accept a subsequent referral to mental health services (33). Depressed lactating women who are recommended to take an antidepressant may concern that the drugs have a possible effect on their infants via breast milk (34). Therefore, understanding the associations between dietary quality and postpartum depression may have some certain meanings.

We found younger mothers tended to have a higher risk of postpartum depression, which was in line with previous researches $(35,36)$. One possible reason was that younger mothers might not be fully prepared for the new maternal role. However, the associations of maternal age and risk of
TABLE 4 | Poisson regression analysis of associations of diet quality and postpartum depression.

\begin{tabular}{lccc}
\hline & PR $(\mathbf{9 5} \% \mathbf{C l})^{\mathbf{a}}$ & $\boldsymbol{P}$ & $\boldsymbol{P}$ for trend \\
\hline HBS & & & \\
Q1 & $1.00($ Ref. $)$ & - & 0.666 \\
Q2 & $0.99(0.93,1.05)$ & 0.666 & - \\
Q3 & $0.99(0.93,1.06)$ & 0.824 & - \\
Q4 & $0.98(0.92,1.05)$ & 0.604 & - \\
LBS & & & \\
Q1 & $1.00($ Ref. & - & -043 \\
Q2 & $1.05(0.98,1.12)$ & 0.154 & - \\
Q3 & $1.02(0.96,1.08)$ & 0.631 & - \\
Q4 & $1.08(1.01,1.15)$ & 0.016 & - \\
DQD & & & - \\
Q1 & $1.00($ Ref. $)$ & - & - \\
Q2 & $0.99(0.93,1.06)$ & 0.805 & - \\
Q3 & $1.00(0.94,1.07)$ & 0.956 & - \\
Q4 & $1.07(1.00,1.14)$ & 0.040 & - \\
\hline
\end{tabular}

${ }^{a}$ Adjusted for maternal age, education, primiparas, and husband or partner support.

depression were still inconclusive. A study has reported that women of advanced maternal age have significantly higher rates of depression than younger women (37). Another study has reported that women's risk for depression increases with age, and age 30 is a critical point (38). Considering the inconsistencies in the existing studies, there is a need for further longitudinal and qualitative studies to understand the associations between maternal age and postpartum depression. We also found less educated women had a higher proportion of postpartum depression, consistent with many studies (39, 
40). Further, primiparas had a higher proportion of postpartum depression. Being a first-time mother was a huge challenge, and it is difficult for primiparas to adapt to their new role $(41,42)$. Lack of husband or partner support was a strong risk factor on postpartum depression $(43,44)$, and we also observed similar results.

We did not observe the associations between family monthly per capita income and postpartum depression, inconsistent with previous studies $(45,46)$. One of the possible explanations is that the data were based on an investigation in urban areas, and the surveyed women have a relatively higher economic level. Future studies could include women from different socioeconomic backgrounds to better understand the associations between family income and postpartum depression among Chinese lactating women. We also did not observe the associations between BMI and postpartum depression. Several biological mechanisms have been hypothesized underlying the association between weight and mental health, such as inflammation, the dysregulation of the hypothalamuspituitary-adrenal axis, and metabolic changes (47), but the association between BMI and postpartum depression are mixed in previous studies $(48,49)$. Since the lactation period is one of substantial weight change and retention for women, the weight information provided by single-point measurement would be limited to some extent, especially for women who had just given birth. Future studies could include information about dynamic changes in body weight during postpartum to understand the associations between BMI and postpartum depression. Although we did not observe the associations between physical activity and postpartum depression, the benefits of physical activity during the postpartum period to postpartum depression have been proved in many types of research, consistent with those for depression among the general population $(50,51)$. Mental and physical health are interdependent, and activities which are started after prior consultation with a specialist and proper instruction are recommended (51).

We found depressed women had less vegetable consumption. Higher vegetable consumption, particularly yellow/orange/red and leafy green vegetables, may help to lower depressive symptoms $(52,53)$, and the possible explanation is certain nutrients found in vegetables, such as vitamins, phytochemicals, minerals, fiber, and amino acids (52). We also found depressed women had more insufficient food variety. Based on our data, a decreased food variety was associated with lower consumption of vegetables, fruit, dairy, and fish and shrimp. All these foods provide our bodies with essential nutrients, such as vitamins, phytochemicals, polyunsaturated fatty acids, and proteins. One study has reported that with an increase in food diversity, the dietary pattern of the individuals could become more similar to the Mediterranean dietary pattern (54), which is inversely associated with many mental health problems, including depression, anxiety, and psychological distress (55). Further, higher LBS and DQD were associated with an increased risk of postpartum depression. Based on DBI calculating method, a score of 0 is in full compliance with the dietary guidelines, and the farther the score is from 0 , the lower the quality of the diet. Higher LBS and DQD indicated more insufficient intake and a more imbalanced diet. The negative association between adherence to dietary guidelines and mental disorders has been reported among Western countries $(56,57)$. This study added related evidence in China, where lifestyle components of lactating women are significantly different from those in Western countries. This favorable association of poor diet quality with postpartum depression may have been attributed to some components of DBI, including vegetables, fruit, dairy, and fish and shrimp, and the cumulatively diverse neuropsychological effects of all diets. One study has reported the inverse association of Alternative Healthy Eating Index (AHEI) with depression and suggested this inverse association has been generated by the cumulative effects of all components of AHEI rather than an individual nutrient or food group (58). The antidepression effect of nutrients is cumulative, and a balanced diet includes multiple components and complex interactions between dietary components and nutrients and their synergistic effects $(56,59)$. Our results highlighted the importance of maintaining the proper dietary balance on the decreased risk of postpartum depression.

Although the current study improves a broader understanding of dietary quality and postpartum depression, several limitations should be considered. First, because the analyses were carried out using cross-sectional data, no causal interpretations can be made. Notably, there may be a bidirectional association in that depressive symptoms and diet quality could be dependent upon one another. More prospective and longitudinal studies are needed. Second, a note of caution was due regarding the extrapolation of our results, in that it was a convenience sample in urban areas of China. Future studies with more representative samples are needed to confirm our results. Third, the DBI score was calculated using a single 24-h recall, which may not reflect usual dietary intake (60). In addition, recall bias cannot be excluded. In our fieldwork, we used a simple semi-quantitative food frequency questionnaire (SFFQ) to obtain participants' average daily food consumption in the past month at the same time. We calculated Spearman's correlation coefficient between 24HDR-derived and SFFQ-derived food groups, and all $P$-values were $<0.01$. The correlation coefficient between 24HDR-derived and SFFQ-derived data may help demonstrate the interrelations between various food groups assessed by different dietary assessment tools. Future studies should further explore and optimize the dietary assessments to obtain more adequate habitual food consumption data $(61,62)$. Fourth, information on some variables, such as marital satisfaction, domestic violence, depression history, and other mental disorders, were not available for our study. Although we have adjusted for a series of potentially confounding covariates, we cannot rule out the possibility of residual confounding.

\section{CONCLUSION}

In our studied population, nearly one-third of lactating women in the first year postpartum suffered from depressive symptoms. 
Depressed women had less vegetable intake and had more insufficient food variety. An imbalanced diet was associated with an increased risk of postpartum depression.

\section{DATA AVAILABILITY STATEMENT}

The raw data supporting the conclusions of this article will be made available by the authors, without undue reservation.

\section{ETHICS STATEMENT}

The studies involving human participants were reviewed and approved by the Medical Ethics Research Board of Peking University (NO. IRB00001052-19045). The patients/participants provided their written informed consent to participate in this study.

\section{AUTHOR CONTRIBUTIONS}

AZ, IS, PW, and YZ: conceptualization. CY, ZR, and JZ: formal analysis. CY, AZ, HL, ZR, and JZ: investigation and methodology.

\section{REFERENCES}

1. Herba CM, Glover V, Ramchandani PG, Rondon MB. Maternal depression and mental health in early childhood: an examination of underlying mechanisms in low-income and middle-income countries. Lancet Psychiatry. (2016) 3:983-92. doi: 10.1016/S2215-0366(16)30148-1

2. Gelaye B, Rondon MB, Araya R, Williams MA. Epidemiology of maternal depression, risk factors, and child outcomes in lowincome and middle-income countries. Lancet Psychiatry. (2016) 3:973-82. doi: 10.1016/S2215-0366(16)30284-X

3. Li Y, Zhao Q, Cross WM, Chen J, Qin C, Sun M. Assessing the quality of mobile applications targeting postpartum depression in China. Int J Mental Health Nurs. (2020) 29:772-85. doi: 10.1111/inm.12713

4. Nisar A, Yin J, Waqas A, Bai X, Wang D, Rahman A, et al. Prevalence of perinatal depression and its determinants in Mainland China: a systematic review and meta-analysis. J Affect Disord. (2020) 277:102237. doi: 10.1016/j.jad.2020.07.046

5. Payne JL, Maguire J. Pathophysiological mechanisms implicated in postpartum depression. Front Neuroendocrinol. (2019) 52:16580. doi: 10.1016/j.yfrne.2018.12.001

6. O’Hara MW, McCabe JE. Postpartum depression: current status and future directions. Annu Rev Clin Psychol. (2013) 9:379-407. doi: 10.1146/annurev-clinpsy-050212-185612

7. Marx W, Moseley G, Berk M, Jacka F. Nutritional psychiatry: the present state of the evidence. Proc Nutr Soc. (2017) 76:42736. doi: 10.1017/S0029665117002026

8. Opie RS, Uldrich AC, Ball K. Maternal postpartum diet and postpartum depression: a systematic review. Matern Child Health J. (2020) 24:96678. doi: 10.1007/s10995-020-02949-9

9. Jacka FN, Pasco JA, Mykletun A, Williams LJ, Hodge AM, O'Reilly SL, et al. Association of Western and traditional diets with depression and anxiety in women. Am J Psychiatry. (2010) 167:305-11. doi: 10.1176/appi.ajp.2009.09060881

10. Nathanson R, Hill B, Skouteris H, Bailey C. Antenatal diet and postpartum depressive symptoms: a prospective study. Midwifery. (2018) 62:6976. doi: 10.1016/j.midw.2018.03.015

11. Pollak MJ, Milte CM, van der Pligt P, Teychenne M. Total physical activity but not diet quality associated with postnatal depressive symptoms amongst women living in socioeconomically disadvantaged neighborhoods. Nutr Res. (2019) 68:54-61. doi: 10.1016/j.nutres.2019.05.009
CY: writing - original draft. AZ, HL, IS, PW, and YZ: writingreview and editing. All authors contributed to the article and approved the submitted version.

\section{FUNDING}

This study was supported by the Inner Mongolia Yili Industrial Group Co. Ltd.

\section{ACKNOWLEDGMENTS}

We thank every participant joining the survey. We also sincerely appreciate the invaluable assistance of our investigation teams from 10 cities.

\section{SUPPLEMENTARY MATERIAL}

The Supplementary Material for this article can be found online at: https://www.frontiersin.org/articles/10.3389/fnut.2021. 705353/full\#supplementary-material

Supplementary Table 1 | The DBI calculating methods in this study.

12. Baskin R, Hill B, Jacka FN, O’Neil A, Skouteris H. The association between diet quality and mental health during the perinatal period. A systematic review. Appetite. (2015) 91:41-7. doi: 10.1016/j.appet.2015. 03.017

13. Zheng L, Sun J, Yu X, Zhang D. Ultra-processed food is positively associated with depressive symptoms among United States adults. Front Nutr. (2020) 7:600449. doi: 10.3389/fnut.2020.600 449

14. Zheng X, Chen J, Xie T, Xia Z, Loo WTY, Lao L, et al. Relationship between Chinese medicine dietary patterns and the incidence of breast cancer in Chinese women in Hong Kong: a retrospective crosssectional survey. Chin Med. (2017) 12:17. doi: 10.1186/s13020-0170138-9

15. Liu Y, Hu J, Chen X, Yu Y, Bai J. Effects of a health education program targeted to Chinese women adhering to their cultural practice of doing the month: a randomized controlled trial. Midwifery. (2020) 90:102796. doi: 10.1016/j.midw.2020.102796

16. Ding G, Niu L, Vinturache A, Zhang J, Lu M, Gao Y, et al. "Doing the month" and postpartum depression among Chinese women: a Shanghai prospective cohort study. Women Birth. (2020) 33:e151-8. doi: 10.1016/j.wombi.2019.04.004

17. Liu N, Mao L, Sun X, Liu L, Yao P, Chen B. The effect of health and nutrition education intervention on women's postpartum beliefs and practices: a randomized controlled trial. BMC Public Health. (2009) 9:45. doi: 10.1186/1471-2458-9-45

18. Huang Y, Wang X, Yang Y, Qu X, Wang A, Huang X, et al. The role of education in maternal depressive symptoms among different ethnic groups: a cross-sectional study in rural western China. J Affect Disord. (2020) 262:35965. doi: 10.1016/j.jad.2019.11.022

19. Lau Y, Wang Y, Yin L, Chan KS, Guo X. Validation of the Mainland Chinese version of the Edinburgh Postnatal Depression Scale in Chengdu mothers. Int J Nurs Stud. (2010) 47:1139-51. doi: 10.1016/j.ijnurstu.2010.02.005

20. Ding Y, Indayati W, Basnet TB, Li F, Luo H, Pan H, et al. Dietary intake in lactating mothers in China 2018: report of a survey. Nutr J. (2020) 19:72. doi: 10.1186/s12937-020-00589-x

21. Yang YY. China Food Composition. 2nd ed. Beijing: Beijing Medical University Press (2009).

22. He D, Qiao Y, Xiong S, Liu S, Ke C, Shen Y. Association between dietary quality and prediabetes based on the diet balance index. Sci Rep. (2020) 10:3190. doi: 10.1038/s41598-020-60153-9 
23. Han A, Sun T, Ming J, Chai L, Liao X. Are the Chinese moving toward a healthy diet? Evidence from Macro data from 1961 to 2017. Int J Environ Res Public Health. (2020) 17:5294. doi: 10.3390/ijerph17155294

24. Yuna H, Yuehui F, Juan X. [Update of the Chinese diet balance index: DBI_16]. Acta Nutrimenta Sinica. (2018) 40:526-30. doi: 10.3969/j.issn.0512-7955.2018.06.002

25. Yuna H, Fengying Z, Xiaoguang Y, Keyou G. [The Chinese diet balance index revised]. Acta Nutrimenta Sinica. (2009) 31:532-6. doi: 10.3321/j.issn:0512-7955.2009.06.003

26. Yuna H, Fengying Z, Keyou G. [Approaching Chinese diet balance index]. J Hyg Res. 2005:208-11. doi: 10.3969/j.issn.1000-8020.2005.02.025

27. Su X, Zhu W, Li N, Sun J, Zhu Y, Liu T, et al. Adjusting DBI2016 to dietary balance index for Chinese maternal women and assessing the association between maternal dietary quality and postpartum weight retention: a longitudinal study. PLoS ONE. (2020) 15:e0237225. doi: 10.1371/journal.pone.0237225

28. Chen C, Lu FC. The guidelines for prevention and control of overweight and obesity in Chinese adults. Biomed Environ Sci. (2004) 17(Suppl.):1-36. doi: 10.1111/j.1365-2028.2008.00991.x

29. Fan M, Lyu J, He P. [Chinese guidelines for data processing and analysis concerning the International Physical Activity Questionnaire]. Zhonghua Liu Xing Bing Xue Za Zhi. (2014) 35:961-4. doi: 10.3760/cma.j.issn.0254-6450.2014.08.019

30. Silverman ME, Reichenberg A, Savitz DA, Cnattingius S, Lichtenstein P, Hultman CM, et al. The risk factors for postpartum depression: a populationbased study. Depress Anxiety. (2017) 34:178-87. doi: 10.1002/da.22597

31. Craig M, Howard L. Postnatal depression. BMJ Clin Evid. (2009) 2009:1407. doi: 10.1016/0140-6736(93)92263-S

32. Gaynes BN, Gavin N, Meltzer-Brody S, Lohr KN, Swinson T, Gartlehner $\mathrm{G}$, et al. Perinatal depression: prevalence, screening accuracy, and screening outcomes. Evid Report Technol Assess. 2005:1-8. doi: 10.1037/e439372005-001

33. Gong W, Jin X, Cheng KK, Caine ED, Lehman R, Xu DR. Chinese women's acceptance and uptake of referral after screening for perinatal depression. Int J Environ Res Public Health. (2020) 17:8686. doi: 10.3390/ijerph17228 686

34. Rampono J, Kristensen JH, Hackett LP, Paech M, Kohan R, Ilett KF. Citalopram and demethylcitalopram in human milk; distribution, excretion and effects in breast fed infants. Br J Clin Pharmacol. (2000) 50:2638. doi: 10.1046/j.1365-2125.2000.00253.x

35. Liu S, Yan Y, Gao X, Xiang S, Sha T, Zeng G, et al. Risk factors for postpartum depression among Chinese women: path model analysis. BMC Pregnancy Childbirth. (2017) 17:133. doi: 10.1186/s12884-017-1320-x

36. Khalifa DS, Glavin K, Bjertness E, Lien L. Determinants of postnatal depression in Sudanese women at 3 months postpartum: a cross-sectional study. BMJ Open. (2016) 6:e009443. doi: 10.1136/bmjopen-2015-009443

37. Muraca GM, Joseph KS. The association between maternal age and depression. J Obstetr Gynaecol Can. (2014) 36:80310. doi: 10.1016/S1701-2163(15)30482-5

38. Luke S, Salihu HM, Alio AP, Mbah AK, Jeffers D, Berry EL, et al. Risk factors for major antenatal depression among low-income African American women. $J$ Womens Health (2002). (2009) 18:1841-6. doi: 10.1089/jwh.2008.1261

39. Matsumura K, Hamazaki K, Tsuchida A, Kasamatsu H, Inadera H. Education level and risk of postpartum depression: results from the Japan Environment and Children's Study (JECS). BMC Psychiatry. (2019) 19:419. doi: 10.1186/s12888-019-2401-3

40. Do TKL, Nguyen TTH, Pham TTH. Postpartum depression and risk factors among vietnamese women. BioMed Res Int. (2018) 2018:4028913. doi: 10.1155/2018/4028913

41. Zhou C, Zheng W, Yuan Q, Zhang B, Chen H, Wang W, et al. Associations between social capital and maternal depression: results from a follow-up study in China. BMC Pregnancy Childbirth. (2018) 18:45. doi: 10.1186/s12884-018-1673-9

42. Wang Q, Zhang Y, Li X, Ye Z, Huang L, Zhang Y, et al. Exploring maternal self-efficacy of first-time mothers among rural-to-urban floating women: a quantitative longitudinal study in China. Int J Environ Res Public Health. (2021) 18:2793. doi: 10.3390/ijerph18062793

43. Li Q, Yang $\mathrm{S}$, Xie $\mathrm{M}$, Wu X, Huang $\mathrm{L}$, Ruan $\mathrm{W}$, et al. Impact of some social and clinical factors on the development of postpartum depression in Chinese women. BMC Pregnancy Childbirth. (2020) 20:226. doi: 10.1186/s12884-020-02906-y

44. Upadhyay RP, Chowdhury R, Aslyeh S, Sarkar K, Singh SK, Sinha B, et al. Postpartum depression in India: a systematic review and meta-analysis. Bull World Health Organization. (2017) 95:706-17c. doi: 10.2471/BLT.17. 192237

45. Leung BM, Letourneau NL, Giesbrecht GF, Ntanda H, Hart M. Predictors of postpartum depression in partnered mothers and fathers from a longitudinal cohort. Community Mental Health J. (2017) 53:420-31. doi: 10.1007/s10597-016-0060-0

46. Gebregziabher NK, Netsereab TB, Fessaha YG, Alaza FA, Ghebrehiwet NK, Sium AH. Prevalence and associated factors of postpartum depression among postpartum mothers in central region, Eritrea: a health facility based survey. BMC Public Health. (2020) 20:1614. doi: 10.1186/s12889-02009676-4

47. Silverman ME, Smith L, Lichtenstein P, Reichenberg A, Sandin $\mathrm{S}$. The association between body mass index and postpartum depression: a population-based study. J Affect Disord. (2018) 240:193-8. doi: 10.1016/j.jad.2018.07.063

48. Sparling TM, Waid JL, Wendt AS, Gabrysch S. Depression among women of reproductive age in rural Bangladesh is linked to food security, diets and nutrition. Public Health Nutr. (2020) 23:660-73. doi: 10.1017/S1368980019003495

49. Carter AS, Baker CW, Brownell KD. Body mass index, eating attitudes, and symptoms of depression and anxiety in pregnancy and the postpartum period. Psychosom Med. (2000) 62:264-70. doi: 10.1097/00006842-20000300000019

50. Dipietro L, Evenson KR, Bloodgood B, Sprow K, Troiano RP, Piercy KL, et al. Benefits of physical activity during pregnancy and postpartum: an umbrella review. Med Sci Sports Exerc. (2019) 51:1292-302. doi: 10.1249/MSS.0000000000001941

51. Kołomańska-Bogucka D, Mazur-Bialy AI. Physical activity and the occurrence of postnatal depression-a systematic review. Medicina. (2019) 55:560. doi: 10.3390/medicina55090560

52. Radavelli-Bagatini S, Anokye R, Bondonno NP, Sim M, Bondonno CP, Stanley MJ, et al. Association of habitual intake of fruits and vegetables with depressive symptoms: the AusDiab study. Eur J Nutr. (2021). doi: 10.1007/s00394-021-02532-0. [Epub ahead of print].

53. Saghafian F, Malmir H, Saneei P, Milajerdi A, Larijani B, Esmaillzadeh A. Fruit and vegetable consumption and risk of depression: accumulative evidence from an updated systematic review and meta-analysis of epidemiological studies. Br J Nutr. (2018) 119:1087-101. doi: 10.1017/S00071145180 00697

54. Poorrezaeian M, Siassi F, Milajerdi A, Qorbani M, Karimi J, Sohrabi-Kabi $\mathrm{R}$, et al. Depression is related to dietary diversity score in women: a crosssectional study from a developing country. Ann Gen Psychiatry. (2017) 16:39. doi: 10.1186/s12991-017-0162-2

55. Sadeghi O, Keshteli AH, Afshar H, Esmaillzadeh A, Adibi P. Adherence to Mediterranean dietary pattern is inversely associated with depression, anxiety and psychological distress. Nutr Neurosci. (2021) 24:248-59. doi: 10.1080/1028415X.2019.1620425

56. Wu PY, Lin MY, Tsai PS. Alternate healthy eating index and risk of depression: a meta-analysis and systemematic review. Nutr Neurosci. (2020) 23:1019. doi: 10.1080/1028415X.2018.1477424

57. Deierlein AL, Ghassabian A, Kahn LG, Afanasyeva Y, Mehta-Lee SS, Brubaker SG, et al. Dietary quality and sociodemographic and health behavior characteristics among pregnant women participating in the New York University children's health and environment study. Front Nutr. (2021) 8:639425. doi: 10.3389/fnut.2021.639425

58. Saneei P, Hajishafiee M, Keshteli AH, Afshar H, Esmaillzadeh A, Adibi P. Adherence to Alternative Healthy Eating Index in relation to depression and anxiety in Iranian adults. Br J Nutr. (2016) 116:335-42. doi: 10.1017/S000711451600 1926

59. Gianfredi V, Koster A, Odone A, Amerio A, Signorelli C, Schaper NC, et al. Associations of dietary patterns with incident depression: the maastricht study. Nutrients. (2021) 131034. doi: 10.3390/nu1303 1034 
60. Verly E Jr, Oliveira DC, Fisberg RM, Marchioni DM. Performance of statistical methods to correct food intake distribution: comparison between observed and estimated usual intake. Br J Nutr. (2016) 116:897903. doi: 10.1017/S0007114516002725

61. Verly E Jr, Castro MA, Fisberg RM, Marchioni DM. Precision of usual food intake estimates according to the percentage of individuals with a second dietary measurement. J Acad Nutr Dietetics. (2012) 112:101520. doi: 10.1016/j.jand.2012.03.028

62. Verly Junior E, Cesar CL, Fisberg RM, Marchioni DM. [Within-person variance of the energy and nutrient intake in adolescents: data adjustment in epidemiological studies]. Revista brasileira de epidemiologia. (2013) 16:1707. doi: 10.1590/S1415-790X2013000100016

Conflict of Interest: IS and HL are employed by Inner Mongolia Yili Industrial Group Co. Ltd. This study received funding from Inner Mongolia Yili Industrial Group Co. Ltd. The funder had the following involvement with the study: conceptualization, investigation, methodology, and writing-review and editing.
The remaining authors declare that the research was conducted in the absence of any commercial or financial relationships that could be construed as a potential conflict of interest.

Publisher's Note: All claims expressed in this article are solely those of the authors and do not necessarily represent those of their affiliated organizations, or those of the publisher, the editors and the reviewers. Any product that may be evaluated in this article, or claim that may be made by its manufacturer, is not guaranteed or endorsed by the publisher.

Copyright (C) 2021 Yang, Zhao, Lan, Ren, Zhang, Szeto, Wang and Zhang. This is an open-access article distributed under the terms of the Creative Commons Attribution License (CC BY). The use, distribution or reproduction in other forums is permitted, provided the original author(s) and the copyright owner(s) are credited and that the original publication in this journal is cited, in accordance with accepted academic practice. No use, distribution or reproduction is permitted which does not comply with these terms. 